\title{
A MULTI CRITERIA RECOMMENDATION MODEL FOR JAUNT
}

\author{
A. Razavi ${ }^{1}$, F. Hosseinali ${ }^{1, *}$ \\ ${ }^{1}$ Department of Surveying Engineering, Faculty of Civil Engineering, Shahid Rajaee Teacher Training University, Tehran, Iran \\ arsalan.razavi92@gmail.com; f.hosseinali@sru.ac.ir
}

KEY WORDS: Recommender Systems, Logistic Regression, Content Based Filtering, Classification, Itinerary, Points of Interest

\begin{abstract}
:
Nowadays, people in most parts of the world always visit, travel and have fun in their cities or other cities, and they spend considerable time and money in their city or in other cities as a tourist. The existence of an intelligent and automated system that can provide the most suitable recreational and cultural offerings at any time and place, with regard to financial capability and time and transport constraints, as well as individual interests and personalization; has always been felt. Recommender systems can be used to suggest suitable recreational options for the user. The main difference between the recommendation model in this study and the previous models is to focus on the short-term planning of a few hours for one day. Previous models were often based on planning a few days a week or days of the month. Also, the cost factor has been considered in this research, which has been less considered in previous models. We used collaborative filtering based on logistic regression to predict whether a type of places is a proper proposition to a user or not. Our case study is about recommending the board game cafés in the city of Kerman, Iran and the result shows that mixed groups between 15 to 30 years old are the best target and our model can predict if board game café is a good suggestion to different users. We used correlation based recommender systems when board game cafes are a proper suggestion for a user and there are at least two options for the user. In case there is no information about the user and his previous rating, popularity based recommender system can be useful. We also used content based recommender systems to give recommendations by having some background information about previous itineraries of a user and his rating to those.
\end{abstract}

\footnotetext{
* Corresponding author
} 


\section{INTRODUCTION}

The enormous amount of information is available over the web, thus the process of making a decision on the large-scale data becomes slow, complicated, and time consuming. Recommender systems alleviates the information overload problem and generates suggestions based on user's preferences, interests, and locations(Nilashi, Ibrahim et al. 2018). RSs originally found success on E-commerce websites to present information on items and products that are likely to be of interest to the user (e.g. films, books, news, webpages, etc.). Lately, they have been increasingly employed in the field of electronic tourism (e-tourism), providing services like trip and activities advisory, lists of points of interest (POIs) that match user preferences, recommendations of tourist packages, etc. (Kabassi 2010) (Werthner and Ricci 2004). Contextual factors such as time, location, and opening and closing hours of POIs should be considered.

Changing context can disappoint the user and significantly affect the travel related decisions (Braunhofer and Ricci 2017). The context-aware travel recommender systems exploits the context of both the user and the item while recommending POI (Bahramian, Ali Abbaspour et al. 2017). Tourists are commonly under inflexible budget restrictions when considering accommodation, meals, means of transport or visits to POIs with entrance fees. Hence, next to the time budget, money budget further constrains the selection of POI visits (Gavalas, Konstantopoulos et al. 2014). Suppose a person enters a new city that has no knowledge of its historical, recreational and sports centers and can only spend a limited time, say 4 hours, and a limited amount of money, and would prefer not to be on the way for more than 1 hour. Without a smart recommender system, he should search the Internet for a list of recreational and cultural centers in the city and encounter a huge amount of information and cannot find accurate information about the money and time required for the proper use of these centers and he will interface with so many places that look the same and it is hard to pick one. Such obstacles cause that in many cases a person will prefer to spend remaining time at the airport or train station or home in fear of losing a flight or recurrent train, but with an intelligent system that can pick the best place among others for the user, individuals can always find a good place to go and this kind of systems can boost tourism in that city as well.

Many RSs are centered around the use of various machine learning and data mining algorithms to predict user evaluations for items, or for learning how to correctly rank items for a user(Ricci, Rokach et al. 2011). By using machine learning algorithms and recommender systems, we can restrict and prioritize options for the user and make suggestions restricted to the user's preferences and choices.

\section{RELATED WORKS}

Internet and mobile devices provide tourists with great opportunities to access tourism information, but the dramatic increase in the number of available tourism choices make it difficult for tourists to choose which option they prefer. Etourism recommender systems are designed to provide suggestions for tourists. Some systems focus on attractions and destinations, while others offer tour plans that include transportation, restaurants and accommodation $(\mathrm{Lu}, \mathrm{Wu}$ et al. 2015). REJA (REstaurants of JAén) hybridizes collaborative filtering and Knowledge based approaches. The recommendations can be provided by the collaborative filtering approach when the system is able to construct a user profile according to the user's ratings. When the system has insufficient information about a user, a case based reasoning approach is executed(Martinez, Rodriguez et al. 2009). CATIS (Context-Aware Tourist Information System) is a context-aware recommender system which recommends tourist accommodation, restaurants and attractions. The context information (e.g., location and wireless device features) is dynamically collected by a context manager. A collection of Web services provided by an application server is used to gather user context information. The recommendations are generated by combining the user query and the user context information from the application server(Pashtan, Blattler et al. 2003). A personalized sightseeing planning system (PSiS), which is used to aid tourists to find a personalized tour plan in the city of Oporto, Portugal, was developed in. To avoid the shortcomings of current recommender systems, such as scalability, sparsity and gray sheep problems, a hybrid recommendation approach was proposed. The proposed hybrid recommendation approach employed collaborative filtering and content based approaches, combined a clustering technique and an associative classification algorithm, and also used fuzzy logic to enhance the quality of recommendations(Lucas, Luz et al. 2013). SigTur/E-Destination was designed to provide personalized recommendations of tourism activities in the region of Tarragona in Spain. To make proper recommendations, the SigTur/E-Destination integrated several types of information and recommendation techniques. The information used in the recommender includes demographic data, details that define the context of the travel, geographical aspects, information provided explicitly by the user and implicit feedback deduced from the interaction of the user with the system. The SigTur/EDestination employs many recommendation techniques, such as the use of stereotypes (standard tourist segments), content based and collaborative filtering techniques, and artificial intelligence tools including automatic clustering algorithms, ontology management, and the definition of new similarity measures between users, based on complex aggregation operators(Moreno, Valls et al. 2013). Traveller is an expert software agent that assists users in the tourism and travel domain. This agent combines collaborative filtering with content-based recommendations and demographic information about customers to suggest package holidays and tours. The combination of techniques in this hybrid approach takes advantage of the positive aspects of each technique and overcomes the difficulties shown by each of them when used in isolation(Schiaffino and Amandi 2009).

\section{METHODOLOGY}

The application of our model is for short journey for pleasure, sometimes including a stay. The word "jaunt" can be used for these types of journey. At first, for better performance of our model, users can enter information such as birthday, gender and city of residence during registering to a desktop (not a mobile) system. Each time using the system, the user enters the amount of money and time that he or she wants to spend on the city. It also asks the user where your current position is and how much time you want to be on the way at most. The more time a user devotes to recreational purposes, the model can add further POIs to the list of possible POIs for jaunt. If the user declares that he is riding a vehicle, the model will reach the POIs according to the average speed of the cars in that city or region, which is usually 5 to 6 times the speed of the ordinary hike. Finally, the user is asked if he or she is currently in favor of a specific type of facility. For example, someone prefers to visit to historical and cultural places, and someone else is interested in spending time and money at the sport facilities. Figure 1 shows the questions that model asks from the user at the 
beginning of the recommendation. Table 1 illustrates an example of gathered information from the users.

What is your ID number? 1002

How much money do you want to spend? (number in dollars) 30

How much time do you have? (number of minutes) 150

What is the maximum time that you want to be on the way? (number of minutes) 30

Do you have car? ( 0 means NO and 1 means YES) 0

What is your Latitude cordinate? (number of cordinate) 35.701248

What is your Longitude cordinate? (number of cordinate) 51.405168

Do you prefer any specific kind of center? (1 means related to food, 2 means related to culture, 3 means related to sport, 4 means related to nature) 0

Are you alone?(1) Are you couple?(2) Are you in a co-gender group?(3 Are you in a mixed group? (4) 4

Figure 1. The questions that model asks from the user

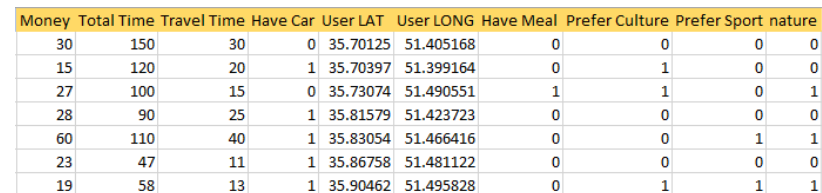

Table 1. Input information from the user at the beginning

Then the system, takes into account this information and other information such as the POIs accessible to the person, the time or money required for each POI, the type of POIs optimal for the person, the day of the week, the openness or closure of the POIs.

The complexities of cities and its streets and phenomena are considerable, so we assumed cities as a regular network to make it simple. The Manhattan distance and the latitude and longitude of points, is used to compute the distance between each two points. Because of the curvature of the earth and the issue that most of the time, there is not a strait way between two points in the cities, we did not use the Euclidian distance on the network. The Manhattan distance between two points is always longer or equal to Euclidian distance, so when our model recommend that a POI is accessible, there is more chance to be a correct suggestion than the suggestion by using the Euclidian distance. We used geopy library to compute this distance in the implemented code in python.

We can calculate the great-circle distance between two points that is the shortest distance over the earth's surface. The distance between point $1\left(\varphi_{1}, \lambda_{1}\right)$ and point $2\left(\varphi_{2}, \lambda_{2}\right)$ can be computed with the following formulas:

$$
\begin{gathered}
\mathrm{a}=\sin ^{2}(\Delta \varphi / 2)+\cos \varphi_{1} * \cos \varphi_{2} * \sin ^{2}(\Delta \lambda / 2) \\
\mathrm{C}=2 * \operatorname{atan} 2(\sqrt{a}, \sqrt{1-a}) \\
\mathrm{D}=\mathrm{R} * \mathrm{C}
\end{gathered}
$$

Where $\varphi$ is latitude, $\lambda$ is longitude, $R$ is earth's radius (mean radius $=6.371 \mathrm{~km}$ )

If the user is at point $\mathrm{A}$ and the POI is at point $\mathrm{B}$, the distance between these two points will be measured as below:

Distance $(\mathrm{A}, \mathrm{B})=\operatorname{Distance}[(\operatorname{Lat} \mathrm{A}$, Long $\mathrm{A}),($ LatA, LongB $)]+$ Distance[(LatA , LongB),(LatB , LongB)]

By multiplying the maximum time on the way and the speed of the user, we can find how far the user can reach. If the user is walking, we will consider his speed $5 \mathrm{~km} / \mathrm{h}$ (the average speed of walking). If the user is driving, we will consider his speed 30 $\mathrm{km} / \mathrm{h}$. Because of using the regular network and the Manhattan distance, the outline of the accessible area for all of the different maximum time on the way is like Figure 3:

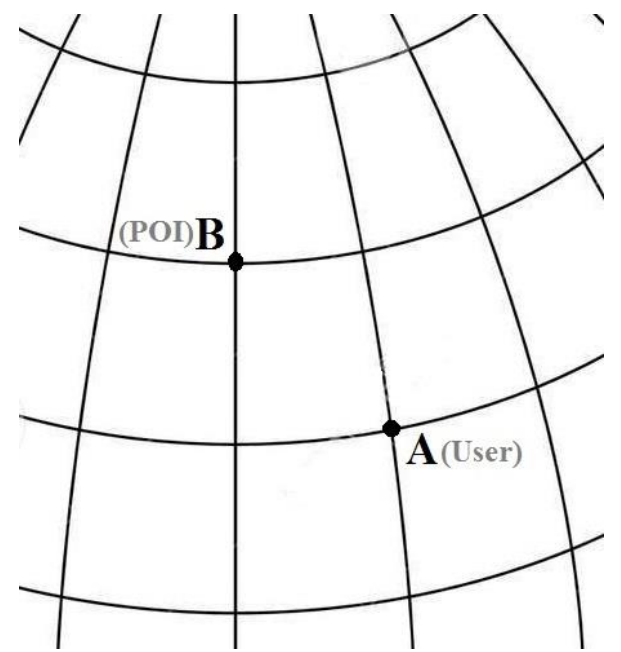

Figure 2. The exaggerated location of two points in the city

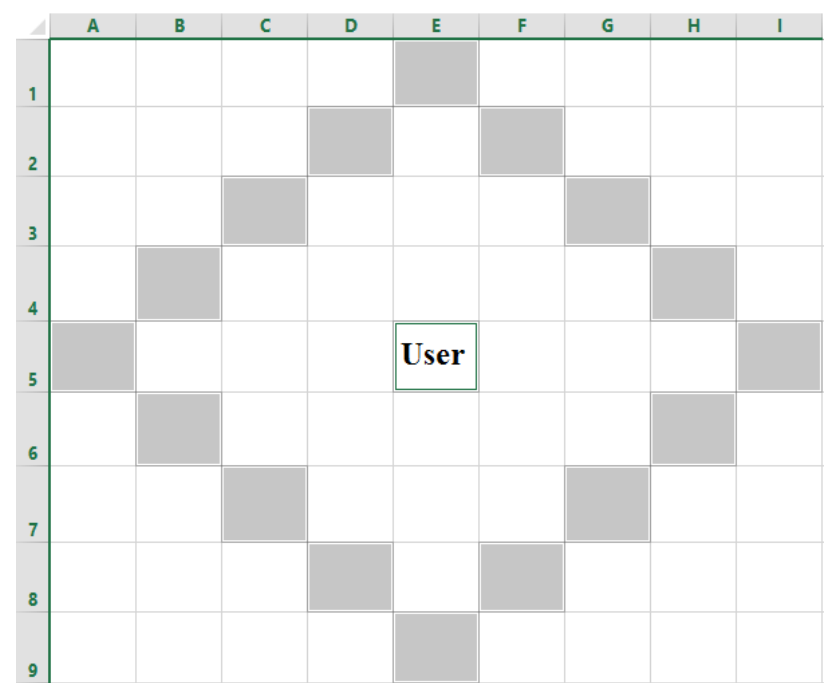

Figure 3. The outline of the accessible area without considering the earth curvature

Higher input maximum time on the way makes a larger accessible area and all the analysis will only perform on the POIs in the accessible area and only the POIs that are within the accessible area can be a possible recommendation for the user. We defined a minimum time and money requirement for each POI in our study. These amount of time and money are required to have a satisfying experience in each POI and the best way to set these number is through asking the staffs of the POIs. If the devoted amount of time and money from the user is lower than the minimum requirement of a POI, that POI will not be recommended to the user. Now we have found the possible POIs for the user, then we use different recommender systems techniques to suggest the best options at first and in the descending order. By using algorithms, the system begins to find and recommend suitable POIs for the person, and if the time and money persists, the system will seek to find a second proposition for the person. For example, the system recommends a person to go to the Board game café A and how much money and time it takes to visit the café (Figure 4), and if enough money and time remains, it will recommend to the user that from which path he can go to Restaurant B. Our developed model is just for very short trips with maximum two destinations. At the end, the user is asked to rate his satisfaction from one to ten or one to five stars from the system's proposed itinerary. 
Board game cafe $\mathrm{A}$ is $100 \mathrm{~m}$ to the right and $275 \mathrm{~m}$ to the up and this place needs 10 to 15 dollars and also 40 to 150 minutes and you are 5 minutes on the way $\wedge$ A

Figure 4 . The offered way for the user by our python code

\subsection{Content Based Filtering}

Content based filtering(Pazzani 1999): The recommendations of those systems depend on content items that the target user has opted for in previous interactions. In particular, various candidate items are compared with items previously rated by the user and the best matching items are recommended.

Content based recommender systems offer users items or locations using the unique profile of each user. Each user profile is gradually made and changed according to his preferences, ratings and number of choices. The recommendation process is based on the similarity between the items. We can measure the similarity and proximity between items based on the content of each item. Content in these systems means classification, tag, or type, and so on. For example, the system for a user called Bahram has so far offered 6 recreational routes, and Bahram gives one point to each of these paths (Table 2). Each recreational route consists of a series of POIs and paths between these POIs. Each of the POIs, locations and routes has different types and attributes, and they can be sports, historical and cultural, natural, For instance, for a user on itinerary 1 takes 20 minutes to walk along a park to get to an athletic club, spending 90 minutes there, then it takes 10 minutes for them to go to a restaurant and spends 40 minutes there eating, then goes to a movie theatre after a 15 -minutes stroll, and spend 80 minutes there for watching a movie. Finally, he gives 4.5 star to this itinerary. The time spent in each of the POIs and routes is recorded according to their type and classification in Table 2.

\begin{tabular}{|l|r|r|r|r|r|r|r|}
\hline & Food & Sport & Nature & Cultural \& History & Cinema \& Art & Score \\
\hline Itinerary 1 & 40 & 90 & 20 & 0 & 80 & 4.5 \\
\hline Itinerary 2 & 30 & 0 & 60 & 0 & 0 & 4.7 \\
\hline Itinerary 3 & 0 & 50 & 0 & 0 & 0 & 3.8 \\
\hline Itinerary 4 & 0 & 0 & 0 & 90 & 30 & 4.9 \\
\hline Itinerary 5 & 60 & 80 & 40 & 0 & 0 & 4 \\
\hline Itinerary 6 & 0 & 60 & 40 & 0 & 0 & 4.2 \\
\hline
\end{tabular}

Table 2. The time spent in different types of POIs and paths

By multiplying the scores given in the time spent on each type of POIs, a new score is obtained for those types of POIs and paths. By repeating the same process for the other proposed itineraries, Table 2 is completed. Then we collect the scores of each type of POIs according to different itineraries to reach total row in Table 3. Then we normalize total row in Table 3, which means that we sum all the scores in total row to find the overall score of all the itineraries and then we divide the scores for each type of the POIs to the overall score of all the itineraries to get to the final coefficient of User profile row in Table 3, which is in fact the profile of our user (Bahram). With a simple look at the user profile, we can see that this user tends to spend time at sports POIs more than other POIs. The profile made for the user can be used to give the next suitable recommendations.

\begin{tabular}{|l|r|r|r|r|r|r|r|}
\hline & Food & Sport & Nature & Cultural \& History & Cinema \& Art & Score \\
\hline Itinerary 1 & 180 & 450 & 90 & 0 & 360 & 4.5 \\
\hline Itinerary 2 & 141 & 0 & 282 & 0 & 0 & 4.7 \\
\hline Itinerary 3 & 0 & 190 & 0 & 0 & 0 & 3.8 \\
\hline Itinerary 4 & 0 & 0 & 0 & 441 & 147 & 4.9 \\
\hline Itinerary 5 & 240 & 320 & 160 & 0 & 0 & 4 \\
\hline Itinerary 6 & 0 & 252 & 168 & 0 & 0 & 4.2 \\
\hline Total & 561 & 1212 & 700 & 441 & 507 & \\
\hline User Profile & 0.16 & 0.35 & 0.2 & 0.13 & 0.15 & \\
\hline
\end{tabular}

Table 3. Overall score for different types of POIs

For example, after performing the initial filterings, 3 paths numbers 7, 8, and 9 are possible for this user. With the user profile and his desire to spend time in any of the different types of recreational POIs, we plan to find the best recommendation from these 3 paths for this user. By multiplying the itinerary matrix in the user profiles vector, the sum of scores is obtained for each itinerary (Equation 5) and the itinerary with the highest score is suggested as the best option for the user.

\begin{tabular}{|l|r|r|r|r|r|r|r|}
\hline & Food & Sport & Nature & Cultural \& History & Cinema \& Art & Score \\
\hline Itinerary 7 & 0 & 0 & 10 & 80 & 90 & 25.9 \\
\hline Itinerary 8 & 30 & 80 & 60 & 0 & 0 & 44.8 \\
\hline Itinerary 9 & 30 & 50 & 0 & 0 & 40 & 28.3 \\
\hline User Profile & 0.16 & 0.35 & 0.2 & 0.13 & 0.15 & \\
\hline
\end{tabular}

Table 4. Using user profile to find the best itinerary

$$
\left[\begin{array}{ccccc}
0 & 0 & 10 & 80 & 90 \\
30 & 80 & 60 & 0 & 0 \\
30 & 50 & 0 & 0 & 40
\end{array}\right] *\left[\begin{array}{c}
0.16 \\
0.35 \\
0.20 \\
0.13 \\
0.15
\end{array}\right]=\left[\begin{array}{l}
25.9 \\
44.8 \\
28.3
\end{array}\right]
$$

Therefore itinerary 8 has the highest score and is the most suitable option, then itinerary 9 and 7 are recommended. If the user chooses and finally gives point to each itinerary, that point and itinerary are also used to update the user's profile

\subsection{Classification Based Collaborative Filtering}

Collaborative filtering (Breese, Heckerman et al. 1998): This type is the most widely used in e-commerce and social media, among others. Target users are recommended items similar to those chosen by other users with similar preferences, therefore users are correlated with each other. A pair of users is correlated on the basis of how common are their individual past selections/ratings.

So far, the logistic regression model has been widely used in many recommendation systems. For example, (Bartz, Murthi et al. 2006)from Yahoo! applied the logistic regression model and collaborative filtering to build an advertising recommendation system. In our model, users are classified into six categories according to their age (Table 5). Also, their gender, marital status, whether the user is alone or spends time with his or her spouse. If a person who is using the system is in a group, he will be asked whether the members of the group are male, female or mixed (Table 6). Preferences of the users is showed in Table 7. Consequently, a vector of individual preferences, ages and others is created. Four example of binary variables that describe each user or group of users are illustrated in vector A, vector B, vector $\mathrm{C}$ and vector $\mathrm{D}$ :

\begin{tabular}{l|l|rrrrrr|r|}
\hline & & A & B & C & D & E & F & G \\
\hline 1 & User & Age $<18$ & $18<$ Age $<29$ & $30<$ Age $<39$ & $40<$ Age $<49$ & $50<$ Age $<59$ & Age $>60$ \\
\hline 2 & A & 0 & 1 & 0 & 0 & 0 & 0 \\
\hline 3 & B & 0 & 1 & 0 & 0 & 0 & 0 \\
\hline 4 & C & 0 & 0 & 1 & 0 & 0 & 0 \\
\hline 5 & D & 1 & 0 & 0 & 0 & 0 & 0 \\
\hline
\end{tabular}

Table 5. Classifying users into categories according to their age 


\begin{tabular}{|c|c|c|c|c|c|c|}
\hline A & $\mathrm{H}$ & 1 & J & $\mathrm{K}$ & L & M \\
\hline 1 User & Gender & Marital Status & Alone & Couple & Cogender Group & Mixed Group \\
\hline A & 1 & c & 0 & 0 & 1 & \\
\hline B & 1 & 0 & 0 & 0 & 1 & 0 \\
\hline c & 0 & 1 & 0 & 0 & 1 & 0 \\
\hline 5 D & 0 & 0 & 1 & 0 & 0 & o \\
\hline
\end{tabular}

Table 6. Gender, marital status and so on

\begin{tabular}{|c|c|c|c|c|c|}
\hline & A & $\mathrm{N}$ & 0 & $\mathrm{P}$ & $Q$ \\
\hline 1 & User & Have Meal & Prefer Culture & Prefer Sport & Prefer Nature \\
\hline 2 & A & 0 & 0 & 1 & 1 \\
\hline 3 & B & 1 & 1 & 0 & 0 \\
\hline 4 & c & 1 & 0 & 1 & 1 \\
\hline 5 & D & 0 & 1 & 1 & 1 \\
\hline
\end{tabular}

Table 7. Preferences of the users

$$
\begin{aligned}
& \mathrm{A}=[0,1,0,0,0,0,1,0,0,0,1,0,0,0,1,1] \\
& \mathrm{B}=[0,1,0,0,0,0,1,0,0,0,1,0,1,1,0,0] \\
& \mathrm{C}=[0,0,1,0,0,0,0,1,0,0,1,0,1,0,1,1] \\
& \mathrm{D}=[1,0,0,0,0,0,0,0,1,0,0,0,0,1,1,1]
\end{aligned}
$$

The Logistic Regression Algorithm is used for the Binary Classification or Dual Classification. The formula is:

$$
P=\frac{1}{1+e^{-\left(\beta_{0}+\beta_{1} x_{1}+\beta_{2} x_{2}+\cdots+\beta_{n} x_{n}\right)}}
$$

Where $\mathrm{P}=$ the probability that $\mathrm{y}=1$

$$
\begin{aligned}
& \beta=\text { the parameters } \\
& \mathrm{x}=\text { the features } \\
& \mathrm{n}=\text { number of features }
\end{aligned}
$$

After dividing the data and completing the learning process, the algorithm give us 0 or 1 response. In this situation, based on user characteristics and user experiences, 0 indicate the location is not suitable, and conversely, 1 indicate the place is suitable.

\subsection{Correlation Based Recommender Systems}

Correlation based recommender systems offer a basic form of collaborative filtering. With correlation based recommender systems, item or places are recommended based on similarities in their user reviews so they do take user preferences into account. These systems use Pearson's R correlation to recommend an item or place that is most similar to the item or place a user has already chosen. In other words, we used correlation to recommend a POI that has a review score that correlates with another POI that a user has already chosen based on the similarity between user ratings.

$$
r=\frac{\sum_{i=1}^{n}\left(X_{i}-\bar{X}\right)\left(Y_{i}-\bar{Y}\right)}{\sqrt{\sum_{i=1}^{n}\left(X_{i}-\bar{X}\right)^{2}} \sqrt{\sum_{i=1}^{n}\left(Y_{i}-\bar{Y}\right)^{2}}}
$$

Where $\mathrm{n}$ is sample size

$X_{i}, Y_{i}$ are the individual sample points indexed with $i$

$\bar{X}$ is the sample mean and analogously for $\bar{Y}$

Having enough number of mutual review score is mandatory and two POIs with only 2 or 3 mutual review score cannot be considered highly correlated. Our model can take several places from the user as the beloved places. So our model can use the correlation of other places with these beloved ones. The places with higher correlation with the beloved places are better options to suggest. We used the folium library in our code to plot the map in Figure 5. We can see that the place with the higher correlation is the first suggestion.

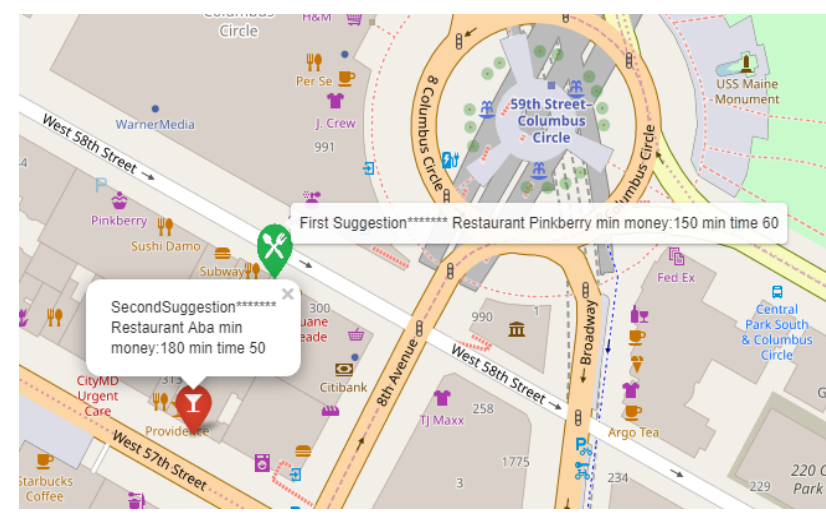

Figure 5. Places that are most similar to the POI a user has already chosen

\subsection{Popularity Based Recommender Systems}

Popularity based recommender systems are the simplest form of collaborative filtering that offer items or places based on how popular those item or places are among other users. These kind of systems do not consider any information or rating about the users, thus these type of systems cannot make personalizes recommendations and considers the place or items with the most number of rating or reviews, the most popular item and suggest that item or places to users. So when there is numbers of places to suggest, these recommender systems offer the place with the most number of rating or reviews. This type of recommender system can be useful to solve the problem of cold start in our recommender model. Cold start problem happens when there is not adequate data for new entries, Cold start problem leads to the inaccurate performance of the collaborative filtering.

\section{IMPLEMENTATION}

Our statistical society is the customers of board game cafés and café goers in the city of Kerman, Iran. We used the data from our ten days of observation and recording them in 3 board game cafés in different areas of the city of Kerman, Iran. In our study, we saved the information of the customers, the information of the age, co-gender or mixed group and other information that were mentioned in table 5,6,7. When the customers were about to leave the board game café, we asked them about the place and if they were satisfied with this place or not. They give one to five star to the place. And we considered 3.5 to 5 stars as a satisfied customer and 1 to 3.4 star as not a good proposition. By dividing the data into $x_{-}$train, $y \_$train, $x_{-}$test and $y_{-}$test, we implemented the training and testing process and the results are in Table 8:

$\begin{array}{rrrr} & \text { precision } & \text { recall } & \text { f1-score } \\ & & & \\ 1 & 0.89 & 0.67 & 0.76 \\ & 0.85 & 0.96 & 0.90 \\ \text { accuracy } & & & 0.86 \\ \text { macro avg } & 0.87 & 0.81 & 0.83 \\ \text { weighted avg } & 0.86 & 0.86 & 0.86\end{array}$

Table 8 . The results of the logistic regression

\section{RESULTS AND CONCLUSIONS}

The result shows that mixed groups between 15 to 30 years old who prefer to have meal are the best target for the board game 
cafés and our model can predict if board game café is a good suggestion to different users. We used the correlation of different places with the beloved places of the user when two places in the accessible area are proper proposition to the user based on logistic regression. The place with higher correlation is the best option. If there is enough information about user rating to different $\mathrm{POI}$, correlation based recommender systems can predict whether a place is a proper proposition to a user or not. Before recommending any POI to the users, our model tries to use logistic regression to find out if it is a rational proposition or not. But in case, there is no information about the user and his previous rating, popularity based recommender system can be useful for a new user in a new city or it can be useful when more than one places are proper suggestions based on logistic regression and we cannot use the correlation based recommender system. It is worth mentioning that this type of recommendation cannot be always a proper choice for example a POI can be popular only among young people and the user is old. By having some background information about previous itineraries of a user and his rating to those, system can build a user profile and suggest some POI to the user even if the user is new to that city or area. To do so, content based recommender systems is used in this paper. Content based recommendation systems are highly efficient, but in some cases they do not work well. For example, if our itinerary has a kind of POI that the user has not visited so far, the system does not offer this path to the user because it does not exist in his profile and the system only recommends itineraries that the user previously used in his profile. For example, if there is an itinerary that only includes musical POIs, this path is not recommended because he has not visited this kind of POIs so far and there is no score for this type of POIs in his profile. These types of problems are solved by using other recommender systems, including collaborative filtering systems.

\section{REFERENCES}

Bahramian, Z., R. Ali Abbaspour and C. Claramunt (2017). "A cold start context-aware recommender system for tour planning using artificial neural network and case based reasoning." Mobile Information Systems 2017.

Bartz, K., V. Murthi and S. Sebastian (2006). Logistic regression and collaborative filtering for sponsored search term recommendation. Second workshop on sponsored search auctions, Citeseer.

Braunhofer, M. and F. Ricci (2017). "Selective contextual information acquisition in travel recommender systems." Information Technology \& Tourism 17(1): 5-29.

Breese, J. S., D. Heckerman and C. Kadie (1998). Empirical analysis of predictive algorithms for collaborative filtering. Proceedings of the Fourteenth conference on Uncertainty in artificial intelligence, Morgan Kaufmann Publishers Inc.

Gavalas, D., C. Konstantopoulos, K. Mastakas and G. Pantziou (2014). "Mobile recommender systems in tourism." Journal of network and computer applications 39: 319-333.

Kabassi, K. (2010). "Personalizing recommendations for tourists." Telematics and Informatics 27(1): 51-66.

Lu, J., D. Wu, M. Mao, W. Wang and G. Zhang (2015). "Recommender system application developments: a survey." Decision Support Systems 74: 12-32.
Lucas, J. P., N. Luz, M. N. Moreno, R. Anacleto, A. A. Figueiredo and C. Martins (2013). "A hybrid recommendation approach for a tourism system." Expert Systems with Applications 40(9): 3532-3550.

Martinez, L., R. M. Rodriguez and M. Espinilla (2009). Reja: a georeferenced hybrid recommender system for restaurants. Proceedings of the 2009 IEEE/WIC/ACM International Joint Conference on Web Intelligence and Intelligent Agent Technology-Volume 03, IEEE Computer Society.

Moreno, A., A. Valls, D. Isern, L. Marin and J. Borràs (2013). "Sigtur/e-destination: ontology-based personalized recommendation of tourism and leisure activities." Engineering Applications of Artificial Intelligence 26(1): 633-651.

Nilashi, M., O. Ibrahim and K. Bagherifard (2018). "A recommender system based on collaborative filtering using ontology and dimensionality reduction techniques." Expert Systems with Applications 92: 507-520.

Pashtan, A., R. Blattler, A. H. Andi and P. Scheuermann (2003). "CATIS: a context-aware tourist information system."

Pazzani, M. J. (1999). "A framework for collaborative, contentbased and demographic filtering." Artificial intelligence review 13(5-6): 393-408.

Ricci, F., L. Rokach and B. Shapira (2011). Introduction to recommender systems handbook. Recommender systems handbook, Springer: 1-35.

Schiaffino, S. and A. Amandi (2009). "Building an expert travel agent as a software agent." Expert Systems with Applications 36(2): 1291-1299.

Werthner, H. and F. Ricci (2004). "E-commerce and tourism." Communications of the ACM 47(12): 101-105. 